\title{
Preclinical testing of the glycogen synthase kinase-3 $\beta$ inhibitor tideglusib for rhabdomyosarcoma
}

\author{
Narendra Bharathy ${ }^{1}$, Matthew N. Svalina ${ }^{1}$, Teagan P. Settelmeyer ${ }^{1}$, Megan M. \\ Cleary $^{1}$, Noah E. Berlow ${ }^{1}$, Susan D. Airhart ${ }^{2}$, Sunny Xiang², James Keck ${ }^{2}$, James B. \\ Hayden ${ }^{3}$, Jack F. Shern ${ }^{4,5}$, Atiya Mansoor6, Melvin Lathara7, Ganapati Srinivasa7, \\ David M. Langenau ${ }^{8,9}$ and Charles Keller ${ }^{1}$ \\ ${ }^{1}$ Children's Cancer Therapy Development Institute, Beaverton, OR 97005, USA \\ ${ }^{2}$ The Jackson Laboratory, Sacramento, CA 95838, USA \\ ${ }^{3}$ Department of Orthopedics and Rehabilitation, Oregon Health \& Science University, Portland, OR 97239, USA \\ ${ }^{4}$ Genetics Branch, Oncogenomics Section, Center for Cancer Research, National Institutes of Health, Bethesda, MD 20892, \\ USA \\ ${ }^{5}$ Pediatric Oncology Branch, Center for Cancer Research, National Institutes of Health, Bethesda, MD 20892, USA \\ ${ }^{6}$ Department of Pathology, Oregon Health \& Science University, Portland, OR 97239, USA \\ ${ }^{7}$ Omics Data Automation, Beaverton, OR 97005, USA \\ ${ }^{8}$ Molecular Pathology, Cancer Center, and Regenerative Medicine, Massachusetts General Hospital, Boston, MA 02114, USA \\ ${ }^{9}$ Harvard Stem Cell Institute, Cambridge, MA 02129, USA \\ Correspondence to: Narendra Bharathy, email: naren@cc-tdi.org \\ Charles Keller, email: charles@cc-tdi.org \\ Keywords: rhabdomyosarcoma, preclinical testing, patient-derived xenograft, GSK3 $\beta$, myodifferentiation \\ Received: December 07, $2016 \quad$ Accepted: June 01,2017 Published: June 16, 2017 \\ Copyright: Bharathy et al. This is an open-access article distributed under the terms of the Creative Commons Attribution License \\ 3.0 (CC BY 3.0), which permits unrestricted use, distribution, and reproduction in any medium, provided the original author and \\ source are credited.
}

\section{ABSTRACT}

Rhabdomyosarcoma (RMS) is the most common childhood soft tissue sarcoma. RMS often arise from myogenic precursors and displays a poorly differentiated skeletal muscle phenotype most closely resembling regenerating muscle. GSK3 $\beta$ is a ubiquitously expressed serine-threonine kinase capable of repressing the terminal myogenic differentiation program in cardiac and skeletal muscle. Recent unbiased chemical screening efforts have prioritized GSK3 $\beta$ inhibitors as inducers of myodifferentiation in RMS, suggesting efficacy as single agents in suppressing growth and promoting self-renewal in zebrafish transgenic embryonal RMS (eRMS) models in vivo. In this study, we tested the irreversible GSK3 $\beta$-inhibitor, tideglusib for in vivo efficacy in patient-derived xenograft models of both alveolar rhabdomyosarcoma (aRMS) and eRMS. Tideglusib had effective on-target pharmacodynamic efficacy, but as a single agent had no effect on tumor progression or myodifferentiation. These results suggest that as monotherapy, GSK3 $\beta$ inhibitors may not be a viable treatment for aRMS or eRMS.

\section{INTRODUCTION}

Rhabdomyosarcoma (RMS) is the most common childhood soft tissue sarcoma and is broadly classified into two histologic subtypes: alveolar rhabdomyosarcoma (aRMS) and embryonal rhabdomyosarcoma (eRMS).
aRMS is a highly aggressive tumor is characterized by the pathogenomic $\mathrm{t}(1: 13)$ or $\mathrm{t}(2: 13)$ translocation resulting in the chimeric gene-fusion product PAX3:FOXO1 or PAX7:FOXO1, respectively [1]. eRMS is often defined as a $R A S$ driven tumor [2-4]. RMS has been shown experimentally to have a myogenic cell-of-origin in 
some model systems and displays a poorly differentiated phenotype with gene expression profiles similar to fetal or regenerating muscle $[5,6]$. Despite the expression of myogenic differentiation-specific transcription factors MYOD1 and myogenin, RMS fails to terminally differentiate [7].

Glycogen synthase kinase $3 \beta(\mathrm{GSK} 3 \beta)$ is a ubiquitously expressed serine-threonine kinase involved in the suppression of skeletal muscle myogenesis and cardiomyocyte hypertrophy via repression of MEF2 transcriptional activity and p38/MAPK signaling [8]. In myoblasts, inhibition of GSK3 $\beta$ induces muscle differentiation $[9,10]$; thus, pharmacologic inhibition of GSK $3 \beta$ has been suggested to be a possible therapeutic avenue towards myodifferentiation in RMS [11].

To this end, recent studies have explored molecularlytargeted therapies that overcome the impaired differentiation in rhabdomyosarcoma [12-15]. A report from our group using unbiased chemical screens prioritized GSK3 $\beta$ inhibitors as inducers of myogenic differentiation in eRMS [11]. In this study, we investigated the expression levels of GSK $3 \alpha$ and GSK3 $\beta$ at the mRNA and protein level in RMS patient samples, RMS cell lines, and normal samples. In addition, we examined the in vivo effect of pharmacologic GSK3 $\beta$ inhibition in aRMS and eRMS.

RNA-Seq data revealed significant differences in the expression of GSK $3 \alpha / \beta$ (and its splice variants) in aRMS and eRMS. We then tested the preclinical efficacy of tideglusib, an irreversible inhibitor of GSK3 $\beta$ [16] in patient-derived xenograft (PDX) models of eRMS and aRMS. In vivo, tideglusib had no effect on tumor progression or myodifferentiation, although pharmacodynamic examination showed efficient reduction in GSK $3 \beta$ mediated phosphorylation of $\beta$-catenin at $\operatorname{Ser}^{33 / 37}$ and $\mathrm{Thr}^{41}$ and consequent stabilization of $\beta$-catenin [17].

\section{RESULTS}

\section{Comparison of GSK3 $\alpha$ and GSK3 $\beta$ expression in normal muscle and RMS cell lines and patient samples}

GSK3 is a serine-threonine kinase that exists as two isoforms encoded by two distinct genes: $G S K 3 \alpha$ and $G S K 3 \beta$ [18]. These isoforms are structurally similar (Figure 1A) but functionally different and exhibit distinct phenotypes [18]. GSK $3 \alpha / \beta$ contain a protein kinase domain with phosphorylation of Tyr $\left(\mathrm{Y}^{279 / 216}\right)$ facilitating its catalytic activity and phosphorylation of $\mathrm{Ser}^{21 / 9}$ inhibiting its own activity $[19,20]$ (Figure 1A). Although GSK $3 \alpha$ exists as GSK $3 \alpha 1 / \alpha 2 / \alpha 3 / \alpha 4$, these variants are rarely specified in literature. GSK $3 \alpha 1 / \alpha 3$ contains an open reading of 483 amino acids each encoding a protein of $51 \mathrm{KDa}$. GSK3 $\alpha 2$ contains an open reading of 401 amino acids encoding a protein of $45 \mathrm{KDa}$ which has not been studied in detail. GSK3 $\alpha 4$ does not form protein [http:// www.ensembl.org/Homo_sapiens/Gene/Summary?db=co re;g=ENSG00000105723;r=19:42230186-42242625].

Similarly, GSK3 $\beta$ exist as spliced variants GSK3 $\beta 1 /$ $\beta 2 / \beta 3 / \beta 4$ (Figure 1A). GSK3 $\beta 1$ is a predominantly expressed spliced variant with GSK3 32 generally accounting for only $15 \%$ of total expression [19]. GSK3 33 / $\beta 4$ does not form protein [http://uswest.ensembl.org/Homo sapiens/Gene/Summary?db=core;g=ENSG00000082701 $; \mathrm{r}=3: 119821323-120094417] . G S K 3 \beta 2$ has 13 additional amino acids inserted in the catalytic (kinase) domain [19] (Figure 1A); however, both GSK3 $\beta 1$ and GSK3 $\beta 2$ have similar phosphorylation patterns at the regulatory sites Ser $^{9}$ and $\mathrm{Tyr}^{216}$. GSK3 $\beta 1 / \beta 2$ exhibits preferential activity towards specific substrates; for example, GSK3 $\beta 2$ has reduced kinase activity towards the microtubule associated protein tau $\left(\mathrm{Ser}^{396}\right)$, phospho-glycogen synthase 2 peptide, CRMP2 (Thr $\left.{ }^{509 / 514}\right)$, CRMP4 $\left(\mathrm{Thr}^{509}\right)$ and Inhibitor-2 $\left(\mathrm{Thr}^{72}\right)$ compared to GSK3 $\beta 1$ [20-21].

To begin the preclinical validation of further preclinical studies of GSK $3 \beta$ inhibition in RMS, we investigated the incidence of GSK $3 \alpha$ and GSK $3 \beta$ expression in RMS. From a published RNA- Seq data [22], we examined GSK3 $\alpha / \beta$ expression in 31 RMS cell lines (17 aRMS, 14 eRMS), 105 RMS patient samples (38 aRMS, 67 eRMS) and 19 normal muscle tissue samples. RMS sample and normal sample expression data are presented as a heatmap partitioned into disease subtype and splice variants in Figure 1B. Overall, RNA expression of GSK $3 \alpha$ and GSK $3 \beta$ statistically differs amongst sample type (RMS patient samples, RMS cell lines, normal muscle samples) and disease type (aRMS, eRMS). GSK $3 \alpha 1$ and GSK $3 \beta 1$ both had high expression across aRMS/eRMS patient samples, cell lines and normal muscle (Figure 1B \& 1C). GSK3 $\alpha 2$ is expressed at increased level by patient samples compared to cell lines and normal muscle, whereas GSK $\alpha 3$ is expressed at lower levels across patient samples and cell lines (Figure 1B \& 1C). GSK3 $\alpha 4$, which has no protein, is expressed at the RNA level in patient samples. Interestingly, GSK $3 \alpha 2$ has no conserved serine amino acid at position twenty one unlike GSK $3 \alpha 1 / \alpha 3$. Phosphorylation of $\operatorname{Ser}^{21}$ inhibit GSK $\alpha 1 / \alpha 3$ activity, raising the question whether GSK $3 \alpha 2$ expression in aRMS and eRMS patients could be related to oncogenesis or progression. Among GSK $3 \beta$, GSK3 $\beta 2$ is expressed at high levels in cell lines and low in patient samples. Similarly, GSK3 34 which has no protein is expressed at high levels in cell lines but at low levels in patient samples. The GSK3 33 splice variant, which has no protein, was expressed at low levels across aRMS/ eRMS patient samples and cell lines (Figure 1B \& 1D). Overall, GSK $3 \alpha 1$ and GSK3 $\beta 1$ that are expressed most consistently at high levels across cell lines/patient samples (Figure 1B \& 1C \& 1D).

To compare co-expression of GSK $3 \alpha$ and GSK3 $\beta$ between RMS patient samples, cell lines, and normal muscle samples for aRMS and eRMS, we measured correlation coefficients of GSK $3 \alpha$ and GSK $3 \beta$ expression 
between samples (Supplementary Figure 1). The coexpression of GSK $3 \alpha$ and GSK3 $\beta$ is statistically different (i.e., unlinked) amongst all sample types in aRMS (cell lines vs. patient tumors $\mathrm{p}=1.38 \mathrm{e}^{-17}$, cell lines vs. normal samples $\mathrm{p}=2.05 \mathrm{e}^{-9}$, patient tumors vs. normal samples $\mathrm{p}=4.04 \mathrm{e}^{-15}$ ) and eRMS (cell lines vs. patient tumors $\mathrm{p}=$ $2.63 \mathrm{e}^{-20}$, cell lines vs. normal samples $\mathrm{p}=1.02 \mathrm{e}^{-5}$, patient tumors vs. normal samples $\mathrm{p}=4.3 \mathrm{e}^{-17}$ ) per Hotelling's $\mathrm{T}^{2}$
A
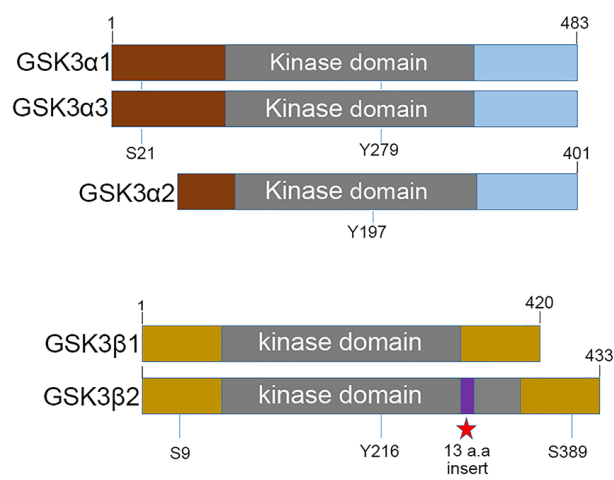

B

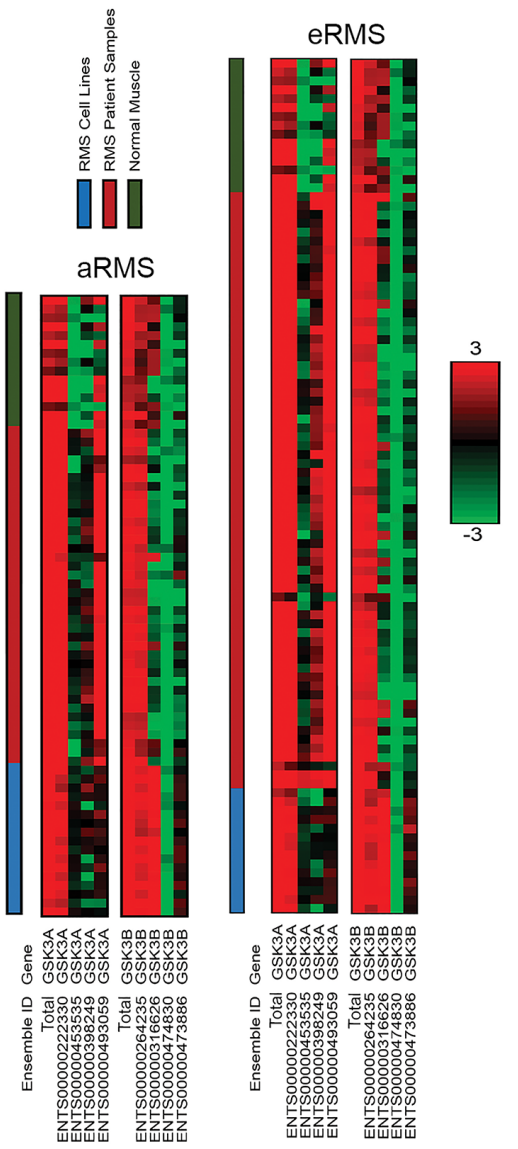

C

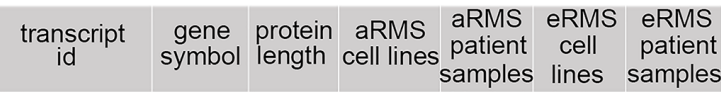

\begin{tabular}{|l|l|l|l|l|l|l|l|l|}
\hline 00000222330 & GSK3a1 483 & high & high & high & high \\
\hline
\end{tabular}

00000398249 GSK3a2 401 low moderate low high

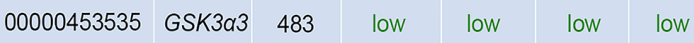

00000493059 GSK3a4 - moderate high moderate high

D

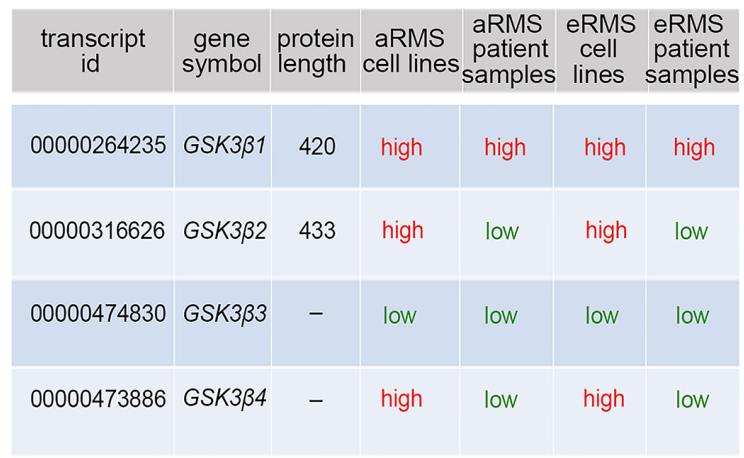

$\mathbf{E}$

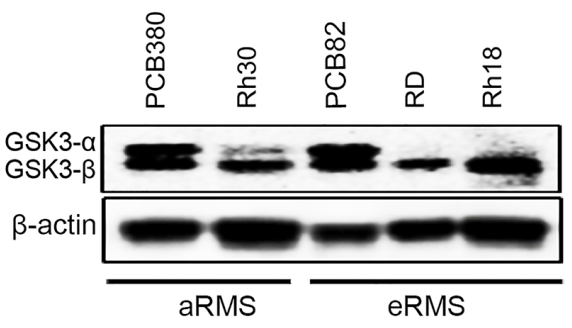

Figure 1: $G S K 3 \alpha$ and $G S K 3 \beta$ expression in RMS cell lines, patient samples, and normal muscle. (A) Schematic representation of full length GSK $3 \alpha 1 / \alpha 2 / \alpha 3$ and GSK3 $\beta 1 / \beta 2$ showing their catalytic domain (kinase), sites of serine (S) and tyrosine (Y) phosphorylation. (B) RNA sequencing was performed on 31 RMS cell lines, 105 RMS patient samples, and 19 normal muscle tissue samples and the resulting $\log 2$-scaled RPKM values for 4 isoforms of $G S K 3 \alpha$ and $G S K 3 \beta$ are shown. Different sample types (RMS cell line, RMS patient sample, normal muscle) are indicated by the color-coded bars at the top of the figure. The heat scale is given on the side, ranging from green (low expression; RPPKM = -3), to black $(\mathrm{RPKM}=0$ ), to red (high expression; RPKM=3).(C \& D) Table showing the different spliced variant of GSK $3 \alpha$ and GSK3 $\beta$ with their respective ensemble ID, gene symbol, protein length (a.a) and their expression across, aRMS, eRMS patient samples and cell lines (color code matching heat map above). (E) Western blotting showing pattern of GSK3 $\alpha / \beta$ expression across aRMS, eRMS, or primary tissue versus cell line samples. GSK3 $\alpha$ and GSK3 $\beta$ have molecular weight of 51 and $47 \mathrm{KDa}$. 
test. MANOVA statistical analysis revealed GSK3 $\alpha$ and GSK3 $\beta$ expression is uncorrelated amongst individual samples and across sample types (GSK3 $\alpha$ samples $\mathrm{p}=2.5 \mathrm{e}^{-8}$, type $\mathrm{p}=2.2 \mathrm{e}^{-7}$, GSK3 $\beta$ samples $\mathrm{p}=1.7 \mathrm{e}^{-13}$, type $\left.\mathrm{p}=2.1 \mathrm{e}^{-14}\right)$ (Figure 1B, Supplementary Figure 1).

To assess if GSK $3 \alpha / \beta$ expression is correlated with clinical outcome, we surveyed a publicly available clinical dataset [23] to analyze the expression of each gene in two patient segments (fusion-positive RMS and fusionnegative RMS) with the corresponding survival of the patient segments (Supplementary Figure 2, Supplementary Table 1). GSK $3 \alpha / \beta$ expression was generated using Affymetrix U95 Gene Chip microarrays, and thus is a unit less measure of gene expression. Only in fusion-positive RMS, GSK expression correlated with statistically significant difference in patient survival, with low versus high GSK3 $\beta$ expression (threshold $=170$ ) showing statistical significance $(p=0.04)$ in patient outcome across 23 patients (Supplementary Figure 2D). Higher expression of GSK $3 \beta$ correlated with improved outcome in the aRMS patients.

To identify a representative xenograft model system, we performed western blot analysis of expression of GSK $3 \alpha / \beta$ in aRMS/eRMS cell lines and PDX tumor cultures (Figure 1E). High GSK $3 \alpha / \beta$ co-expression was seen in aRMS primary tumor culture PCB380 and eRMS primary tumor culture PCB82 in comparison to aRMS cell lines Rh30 and eRMS cell lines RD and Rh18. RNASeq data (GEO Accession number GSE100427) revealed higher expression of GSK3 $\alpha 1 / \beta 1$ compared to other splice variants in PCB380/82 (Supplementary Tables $2 \& 3 \&$ 4 \& 5). Thus, PDX models PCB 380 and PCB 82 were selected as model systems for further studies.

\section{Tideglusib shows pharmacological efficacy in vitro}

To test the pharmacological activity of tideglusib against GSK3 $\beta$, PDX derived cell cultures PCB82 (eRMS) and PCB380 (aRMS) were treated with tideglusib at its previously reported enzyme IC50 of $60 \mathrm{nM}$, which has been shown to bring irreversible inhibition of kinase activity of GSK3 $\beta$ (in cell free assay) [16]. After twenty minutes, cells were harvested and analyzed for the GSK3 $\beta$ mediated phosphorylation status of $\beta$-catenin, which was reduced substantially upon treatment. A corresponding increase in total levels of $\beta$-catenin was also observed which indicated the efficacy of tideglusib in blocking GSK3 $\beta$ activity (17) (Figure 2A \& 2B).

\section{Tideglusib shows no in vivo effect on survival or myodifferentiation}

We performed preclinical testing of tideglusib in PDX mouse models of aRMS (PCB380) and eRMS (PCB82). The maximum tolerated dose (MTD) of tideglusib was determined experimentally and found to be $200 \mathrm{mg} / \mathrm{kg}$ (data not shown). No significant toxicity (weight loss, activity change) was observed at this dose.

Tumor-bearing mice were treated with $200 \mathrm{mg} / \mathrm{kg}$ of tideglusib daily by oral gavage. Kaplan-Meier survival analysis showed no significant differences in survival between Group 1 and Group 2 (PCB82 eRMS treated with vehicle vs tideglusib, $\mathrm{p}=0.972$ ) or Group 3 and Group 4 (PCB380 aRMS treated with vehicle vs tideglusib, $\mathrm{p}=0.612$ ) (Figure 2C). Pharmacodymanic analysis showed significant reduction in the GSK3 $\beta$ mediated phosphorylation of $\beta$-catenin and an increase in total $\beta$-catenin in tumor lysates (Figure 2D \& 2E) in both eRMS ( $=0.038)$ (Figure 2F; upper panel) and aRMS model $(\mathrm{p}=0.024)$ (Figure $2 \mathrm{~F}$; lower panel) demonstrating that tideglusib treatment inhibits catalytic activity of GSK3 $\beta$ but does not improve survival.

We also examined the effect of tideglusib on myodifferentiation. Immunohistochemistry on treatment and control groups from both aRMS and eRMS PDX model did not exhibit any rhabdomyoblasts (Supplementary Figure $3 \&$ Supplementary Table 6). Western blot analysis of tideglusib-treated aRMS and eRMS PDX tumor protein lysates showed no induction of myodifferentiation (via myosin heavy chain expression) in treated versus control groups. Differentiated human skeletal myoblasts (HSMM) were used as a positive control (Figure 2G \& 2H). Following tideglusib treatment, myogenin was downregulated in eRMS (Figure 2G), and aRMS (Figure 2H). Overall, tideglusib had no effect on survival or myodifferentiation.

\section{DISCUSSION}

To our knowledge this is the first study 1) to analyze the expression of GSK $3 \alpha / \beta$ and its splice variants across a broad collection of RMS cell lines and patient samples in comparison to normal muscle samples, and 2) to examine pharmacological inhibition of GSK3 $\beta$ in PDX RMS models.

Tideglusib, an irreversible inhibitor of GSK3 $\beta$, was tested for preclinical efficacy against RMS PDX models. Tideglusib displayed pharmacokinetic potency in reducing GSK3 $\beta$-mediated phosphorylation of phospho- $\beta$-catenin and consequent increase in total $\beta$-catenin in vitro for primary tumor cultures with demonstrated over-expression of GSK3 $\alpha$ and GSK3 $\beta$. Despite this on-target efficacy, tideglusib tested at the highest dosage $(200 \mathrm{mg} / \mathrm{kg})$ had no effect on in vivo tumor growth or myodifferentiation in PDX models of aRMS or eRMS. Unexpectedly, tideglusib treatment showed reduction in myogenin in aRMS/eRMS PDX tumor. This is surprising as recent study shows that myogenin function in aRMS is disrupted by sustained GSK $3 \beta$ kinase activity contributing to the undifferentiated, proliferative phenotype [13]. However, the difference in observation could be attributed to the fact that this study is limited to one aRMS cell line, Rh30. Our studies of 
A

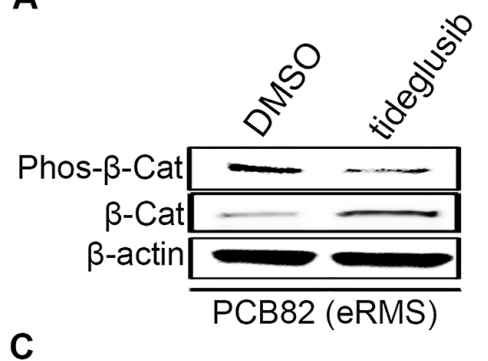

B

D

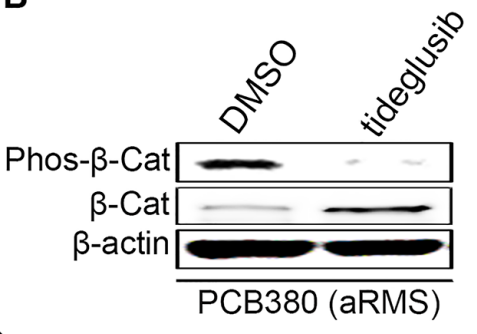

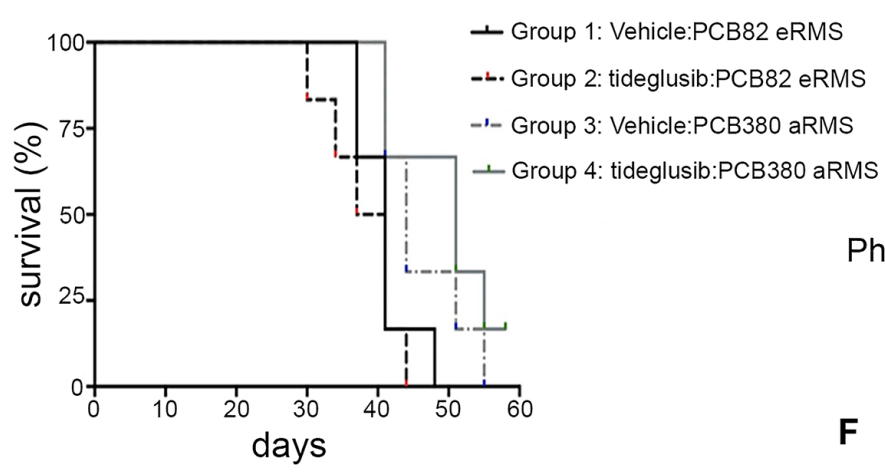

E

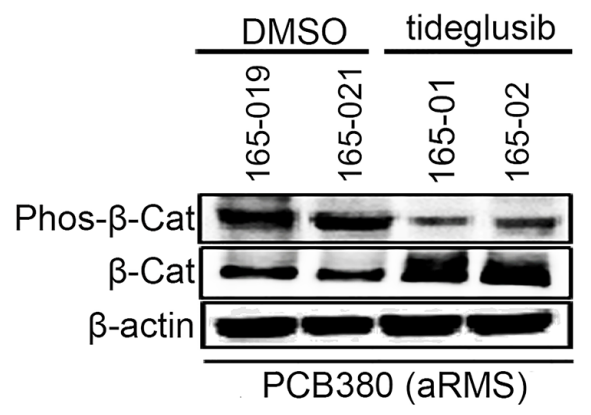

G

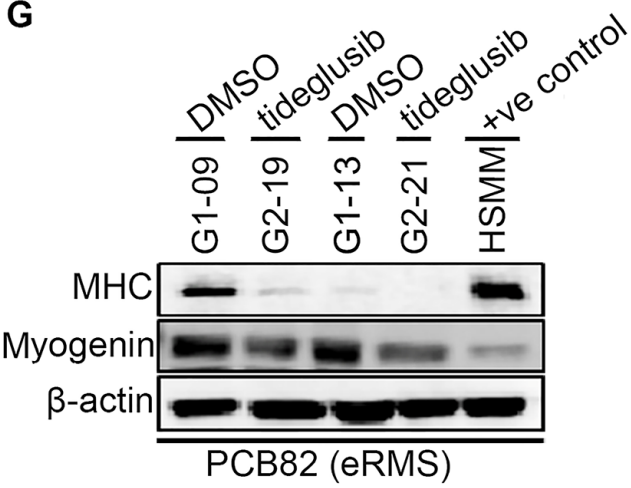

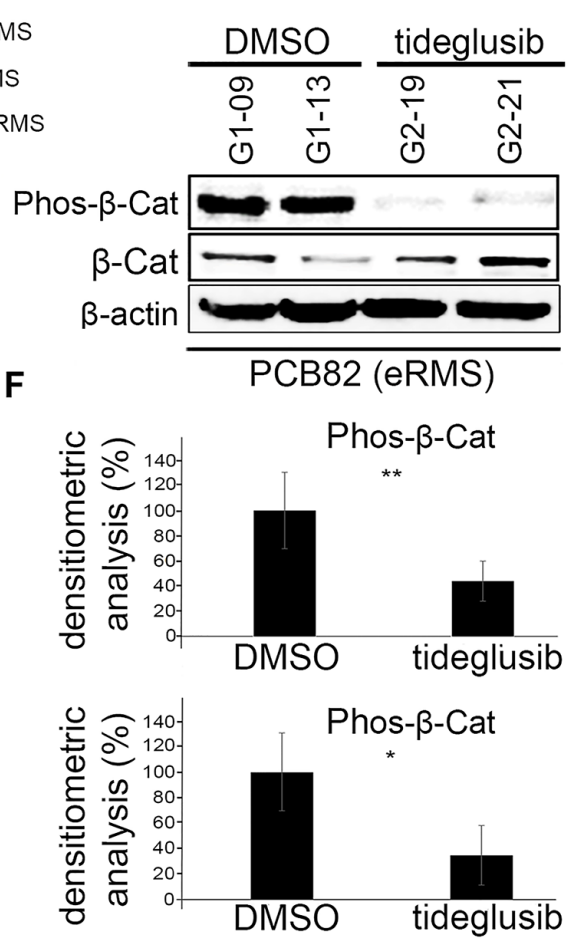

H

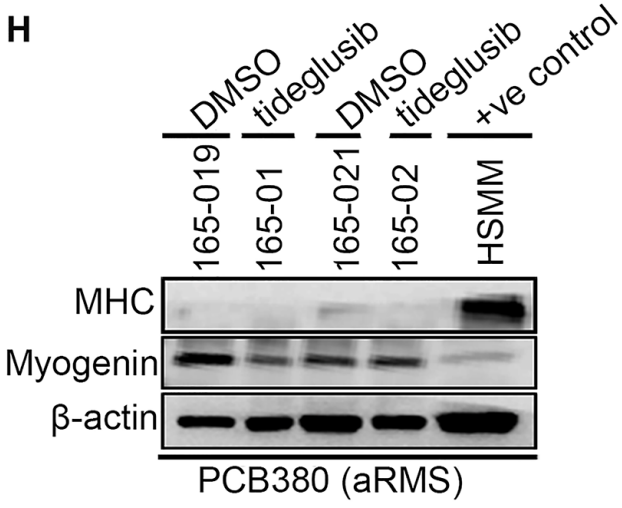

Figure 2: Effects of tideglusib on tumor growth, myodifferentiation in vivo. (A \& B) Western blotting of vehicle and tideglusib treated human PDX derived primary culture (PCB82 and PCB380) for detection of GSK-3 $\beta$ mediated phosphorylation of $\beta$-catenin which showed reduction and an increase in total $\beta$-catenin. (C) Kaplan-Meier curve showing eRMS (PCB82) and aRMS mice (PCB380) treated with $200 \mathrm{mg} / \mathrm{kg}$ tideglusib via oral gavage daily experienced no effect on survival. (D \& E) Western blotting of vehicle and tideglusib treated human PDX tumors (PCB82 and PCB380) for detection of GSK3 $\beta$ mediated phosphorylation of $\beta$-catenin which showed reduction and an increase in total $\beta$-catenin. (F) Densitometric analysis shows the reduction in phos- $\beta$-catenin upon tideglusib treatment in eRMS (PCB82) (upper panel) and aRMS (PCB380) (lower panel) to be statistically significant $\left({ }^{*} \mathrm{p}>0.05 ;{ }^{* *} \mathrm{p}>0.001\right.$ ). Error bars represent mean \pm S.D. (G \& H) Western blotting of vehicle and tideglusib treated human PDX derived primary culture (PCB82 and PCB380) for detection of myogenin and myosin heavy chain (MHC). Differentiated HSMM used as a positive control. 
GSK $3 \alpha / \beta$ isoform expression suggest that cell lines have a skewed isoform and splice variant expression compared to primary tumor tissue, which is important to take into account. Our findings also suggests that GSK $3 \beta$ is not the optimal therapeutic target for single-agent treatment of aRMS/eRMS. Yet while tideglusib may be of limited value as a monotherapy in RMS, future studies could explore combination therapies with chemotherapy agents. Furthermore, since GSK3 is regulated by multiple pathways, new roles of GSK $3 \alpha$ and GSK $3 \beta$, under nonhomeostatic 'stress' conditions may be uncovered.

\section{MATERIALS AND METHODS}

\section{Cell culture}

aRMS cell line Rh30, eRMS cell lines RD and Rh18, and PDX cultures PCB380 and PCB82 were cultured in growth medium (GM) RPMI 1640 (11875-093; Thermo Fisher Scientific, Waltham, MA, USA) supplemented with 10\% fetal bovine Serum (FBS) (26140079; Thermo Fisher Scientific) and 1\% penicillin/Streptomycin (15140122; Thermo Fisher Scientific). Primary human skeletal myoblasts (HSMM) (CC-2580; Lonza Inc, Allendale, NJ, USA) were cultured in GM (SKBM-2 CC-3244; Lonza Inc) supplemented with $10 \%$ FBS and $1 \%$ penicillin/ streptomycin. HSMM was differentiated until day 4 in differentiation medium DM: F-12 (11320033; Thermo Fisher Scientific). All cells were incubated at $37^{\circ} \mathrm{c}$ and $5 \% \mathrm{CO}_{2}$. aRMS and eRMS cell lines were authenticated by short tandem repeat (STR) analysis (Biosynthesis, Lewisville, TX, USA).

\section{Tideglusib cell treatments}

Tideglusib, an irreversible inhibitor of GSK3 $\beta$ was purchased from SelleckChem (S2823, Houston, TX, USA). aRMS and eRMS PDX primary tumor cell cultures were treated with tideglusib at its IC50 for GSK3 $\beta$ (60 nM) [16].

\section{Patient-derived xenograft models}

RMS samples were collected from patients undergoing planned surgical resection or research-autopsy enrolled in the bio-banking and model development program Childhood Cancer Registry for Familial and Sporadic Tumors (CuRe-FAST). All patients provided informed consent; patient data and clinical and pathologic findings are maintained in a de-identified database. All aspects of the study were reviewed and approved by the Oregon Health \& Science University Institutional Review Board and the Children's Cancer Therapy Development Institute Institutional Review Board. The eRMS PDX PCB82 (TM00360) was recently described [24], and the Pax3:Foxo1+ aRMS PDX model PCB380 (TM01165) was similarly established from an untreated, surgically-resected soleus tumor from a 2-year-old female. To establish each
PDX model, NSG (NOD.Cg-Prkdc $c^{\text {scid }} I L 2 r g^{\text {tm } 1 \text { Wjl/SzJ }}$ ) mice were obtained by The Jackson Laboratory. Tumor fragments were obtained from the aforementioned patients and implanted into the rear flanks of recipient female NSG (JAX \# 5557) mice using a trochar. Once tumors reached 1cc, they were collected and fragmented for serial transplantation in NSG mice to create low-passage cohorts for future studies. All studies were done with the approval of The Jackson Laboratory IACUC. For tissue culture work, PCB380 and PCB82 were authenticated by analysing for the expression of myogenin and lack of MHC expression.

\section{Generation of RNA-Seq data} [22].

RNA sequencing data has been previously described

\section{Western blot}

To prepare protein lysate, tumors and PDX cell lines PCB82 and PCB380 treated with vehicle (control) and tideglusib were lysed in radio immunoprecipitation (RIPA) buffer (89901, Thermo Fisher Scientific) containing both protease and phosphatase inhibitors (Sigma Aldrich, St. Louis, MO). Lysates were homogenized and clarified by centrifugation at $14,000 \mathrm{rpm}$ for 10 minutes. Thirty $\mu \mathrm{g}$ of protein were electrophoresed in 7.5-10\% mini protean polyacrylamide gel (4561024, Bio-Rad, Hercules, CA, USA) transferred to PVDF membranes (1620255, Bio-Rad) for immunoblot analysis with anti-GSK $3 \alpha / \beta$ (sc-7291, Santa Cruz Biotechnology, Dallas, TX, USA) (the monoclonal antibody used is raised against the amino acid 1-420 representing the full length GSK3 $\beta$ ), antiPhos- $\beta$-Catenin (9561, Cell Signaling, Danvers, MA, USA), anti- $\beta$-Catenin (9562, Cell Signaling), anti-MHC (MAB4470, R\&D systems, Minneapolis, MN, USA), anti-myogenin (sc-576, Santa Cruz Biotechnology), anti$\beta$-actin (ab8227, Abcam, San Francisco, CA, USA). Blots were developed using FluorChem Q system (92-14095-00, protein simple, San Jose, CA, USA).

\section{Xenograft studies}

Tideglusib was dissolved in 30\% PEG400/0.5\% Tween $80 / 5 \%$ propylene glycol, $30 \mathrm{mg} / \mathrm{mL}$ and formulated for in vivo testing in 26\% PEG400 (Polyethylene Glycol 400), $15 \%$ Cremophor EL. The MTD of tideglusib was determined experimentally. Mice ( $\mathrm{n}=3$ per arm) were randomized to 100 , 150 , or $200 \mathrm{mg} / \mathrm{kg}$ treatment arms. Mice were dosed by oral gavage daily for 21 days. Clinical observations of general behavior, mobility, and weight loss were performed daily. MTD was defined as the dosage that causes a mean weight loss of $>20 \%$ as a group or a body condition of $<2$ for one or more animals in a group. For PDX preclinical efficacy studies, forty-eight 5-6 week old NSG mice were obtained from The Jackson Laboratories. Twenty-four mice were trochar implanted with a passage 1-3 PDX fragment in the 
right hind flank to generate study mice. Mice were monitored twice weekly for clinical observations and body weight. Tumor-bearing mice were randomized into treatment cohorts of $n=6$ mice per group so that the mean cohort tumor volume was 200-300 cc. Mice were treated with vehicle or tideglusib administered by oral gavage at a dose of $200 \mathrm{mg} / \mathrm{kg}$ daily until tumors reached $2000 \mathrm{cc}$ or the condition of the mice precluded ongoing treatment.

\section{Statistical analysis}

The tumor endpoint volumes for time-to-event (TTE) analysis were set at $1500 \mathrm{~mm}^{3}$ and $2000 \mathrm{~mm}^{3}$, in the tideglusib study. TTE was defined in days by selecting the day in which the tumor volume equaled or surpassed 1500 $\mathrm{mm}^{3}$ and $2000 \mathrm{~mm}^{3}$. Animals that did not reach endpoint volume were assigned a TTE of 58 days. Comparisons were analyzed by One-way Anova with Bonferroni's post-test. The Kaplan-Meier survival plot represents the percentage of animals surviving at different time points during the study. These percentages were generated from the TTE data using GraphPad Prism 6.0 software. Survival curve comparisons were analyzed by the Mantel-Cox and Gehan-BreslowWilcox tests (95\% CI) through Graph Pad Prism software. For densitometric analysis, significance was determined by a one-tailed Student's t test and $p$ values of $<0.05$ were considered to be statistically significant. Statistical significance were set at $* \mathrm{P}<0.05$ and $* * \mathrm{P}<0.01$. Error bars indicate mean \pm standard deviation (SD).

\section{Author contributions}

NB, MNS, SDA, JK, DML and CK designed the study; NB, MNS, TPS, MMC, AM, SX performed experiments; NEB, JFS, ML, GS performed computational analysis; NB, NEB, MNS and CK analyzed and interpreted data; JBH provided samples; NB, MNS, TPS, NEB, JFS, DML and CK wrote the manuscript; $\mathrm{CK}$ directed studies.

\section{CONFLICTS OF INTEREST}

The authors declare that they have no conflicts of interest.

\section{GRANT SUPPORT}

This study was funded by SARC SPORE Developmental Research Program Grant, 1U54CA168512-01.

\section{REFERENCES}

1. Barr FG. Gene fusions involving PAX and FOX family members in alveolar rhabdomyosarcoma. Oncogene. 2001; 20:5736-46.
2. Langenau DM, Keefe MD, Storer NY, Guyon JR, Kutok JL, Le X, Goessling W, Neuberg DS, Kunkel LM, Zon LI. Effect of RAS on the genesis of embryonal rhabdomyosarcoma. Genes Dev. 2007; 21:1382-95.

3. Stern DF, Roberts AB, Roche NS, Sporn MB, Weinberg RA. Differential responsiveness of myc- and ras-transfected cells to growth factors: selective stimulation of myc transfected cells by epidermal growth factor. Mol Cell Biol. 1986; 6:870-7.

4. Chen X, Stewart E, Shelat AA, Qu C, Bahrami A, Hatley M, Wu G, Bradley C, McEvoy J, Pappo A, Spunt S, Valentine $\mathrm{MB}$, Valentine $\mathrm{V}$ et al. Targeting oxidative stress in embryonal rhabdomyosarcoma. Cancer Cell. 2013; 24:710-24.

5. Rubin BP, Nishijo K, Chen HI, Yi X, Schuetze DP, Pal R, Prajapati SI, Abraham J, Arenkiel BR, Chen QR, Davis S, McCleish AT, Capecchi MR et al. Evidence for an unanticipated relationship between undifferentiated pleomorphic sarcoma and embryonal rhabdomyosarcoma. Cancer Cell. 2011; 19:177-191.

6. Tonin PN, Scrable H, Shimada H, Cavenee WK. Musclespecific gene expression in rhabdomyosarcomas and stages of human fetal skeletal muscle development. Cancer Res. 1991; 51:5100-6.

7. Tapscott SJ, Thayer MJ, Weintraub H. Deficiency in rhabdomyosarcomas of a factor required for MyoD activity and myogenesis. Science. 1993; 259:1450-3.

8. Dionyssiou MG, Nowacki NB, Hashemi S, Zhao J, Kerr A, Tsushima RG, Mc Dermott JC. Cross-talk between glycogen synthase kinase 3beta (GSK3beta) and p38MAPK regulates myocyte enhancer factor 2 (MEF2) activity in skeletal and cardiac muscle. J Mol Cell Cardiol. 2013; 54:35-44.

9. Brack AS, Conboy IM, Conboy MJ, Shen J, Rando TA. A temporal switch from notch to Wnt signaling in muscle stem cells is necessary for normal adult myogenesis. Cell Stem Cell. 2008; 2:50-9.

10. Rochat A, Fernandez A, Vandromme M, Moles JP, Bouschet T, Carnac G, Lamb NJl. Insulin and wnt1 pathways cooperate to induce reserve cell activation in differentiation and myotube hypertrophy. Mol Biol Cell. 2004; 15:4544-5.

11. Chen EY, DeRan MT, Ignatius MS, Grandinetti KB, Clagg R, McCarthy KM, Lobbardi RM, Brockmann J, Keller C, Wu X, Langenau DM. Glycogen synthase kinase 3 inhibitors induce the canonical WNT/beta-catenin pathway to suppress growth and self-renewal in embryonal rhabdomyosarcoma. Proc Natl Acad Sci U S A. 2014; 111:5349-54.

12. Annavarapu SR, Cialfi S, Dominici C, Kokai GK, Uccini S, Ceccarelli S, McDowell HP, Helliwell TR. Characterization of Wnt/beta-catenin signaling in rhabdomyosarcoma. Lab Invest. 2013; 93:1090-9.

13. Dionyssiou MG, Ehyai S, Avrutin E, Connor MK, McDermott JC. Glycogen synthase kinase 3beta represses 
MYOGENIN function in alveolar rhabdomyosarcoma. Cell Death Dis. 2014; 5:e1094.

14. Tremblay AM, Missiaglia E, Galli GG, Hettmer S, Urcia R, Carrara M, Judson RN, Thway K, Nadal G, Selfe JL, Murray G, Calogero RA, De Bari C, et al. The Hippo transducer YAP1 transforms activated satellite cells and is a potent effector of embryonal rhabdomyosarcoma formation. Cancer Cell. 2014; 26:273-87.

15. Zeng FY, Dong H, Cui J, Liu L, Chen T. Glycogen synthase kinase 3 regulates PAX3-FKHR-mediated cell proliferation in human alveolar rhabdomyosarcoma cells. Biochem Biophys Res Commun. 2010; 391:1049-55.

16. Domínguez JM, Fuertes A, Orozco L, del Monte-Millán M, Delgado E, Medina M. Evidence for irreversible inhibition of glycogen synthase kinase-3 $\beta$ by tideglusib. J Biol Chem. 2012; 287:893-904.

17. Yost C, Torres M, Miller JR, Huang E, Kimelman D, Moon RT. The axis-inducing activity, stability, and subcellular distribution of beta-catenin is regulated in Xenopus embryos by glycogen synthase kinase 3. Genes Dev. 1996; 10:1443-54.

18. Woodgett JR. Molecular cloning and expression of glycogen synthase kinase-3/factor A. EMBO J. 1990; 9:2431-8.

19. Mukai F, Ishiguro K, Sano Y, Fujita SC. Alternative splicing isoform of tau protein kinase I/glycogen synthase kinase 3beta. J Neurochem. 2002; 81:1073-83.
20. Doble BW, Woodgett JR. GSK-3: tricks of the trade for a multi-tasking kinase. J Cell Sci. 2003; 116:1175-86.

21. Soutar MP, Kim WY, Williamson R, Peggie M, Hastie CJ, McLauchlan H, Snider WD, Gordon-Weeks PR, Sutherland C. Evidence that glycogen synthase kinase-3 isoforms have distinct substrate preference in the brain. J Neurochem. 2010; 115:974-83.

22. Shern JF, Chen L, Chmielecki J, Wei JS, Patidar R, Rosenberg M, Ambrogio L, Auclair D, Wang J, Song YK, Tolman C, Hurd L, Liao H, et al. Comprehensivegenomic analysis of rhabdomyosarcoma reveals a landscape of alterations affecting a common genetic axis in fusionpositive and fusion-negative tumors. Cancer Discov. 2014; 4:216-31.

23. Blandford MC, Barr FG, Lynch JC, Randall RL, Qualman SJ, Keller C. Rhabdomyosarcomas utilize developmental, myogenic growth factors for disease advantage: a report from the Children's Oncology Group. Pediatr Blood Cancer. 2006; 46:329-38.

24. Hooper JE, Cantor EL, Ehlen MS, Banerjee A, Malempati S, Stenzel P, Woltjer RL, Gandour-Edwards R, Goodwin NC, Yang Y, Kaur P, Bult CJ, Airhart SD, et al. A patientderived xenograft model of parameningeal embryonal rhabdomyosarcoma for preclinical studies. Sarcoma. 2015; 2015:826124. 